\title{
Detection, five-year monitoring, and management of a nonprogressive lesion of pre-eruptive intracoronal radiolucency
}

\author{
Jawza H. Alfarraj*, Muhammad A. Alhelal \\ Pediatric Dentistry Department, King Faisal Specialist Hospital and Research, Riyadh Central 14215, Saudi Arabia
}

\begin{abstract}
Objectives: To report a case of pre-eruptive intracoronal radiolucency (PEIR) in the right mandibular second permanent molar of a young female patient, and to promote awareness of early detection of PEIR. It is a condition where unerupted teeth exhibit radiographic caries like radiolucency within the dentin and underneath the enamel.

Study design: This report describes an incidental finding of an asymptomatic REIR seen in a panoramic radiograph. When the tooth was partially erupted, it was sealed to prevent bacterial colonization. After the tooth became fully erupted, conservative restorative treatment was performed and the patient was followed up for another year.
\end{abstract}

Results: The detected lesion was monitored for five years and found to be nonprogressive.

Conclusion: It is critical to monitor the progression of asymptomatic PEIR lesions by periodic radiographic examination. For the nonprogressive type of PEIR, we support following a conservative approach by monitoring until the tooth is fully erupted.

\section{Introduction}

Pre-eruptive intracoronal radiolucency (PEIR) is a condition where unerupted teeth demonstrate radiographic coronal radiolucency within the dentin layer and underneath the dentino-enamel junction (DEJ) $[1,2]$. Most PEIR lesions are located in the middle of the crown. However, a smaller percentage are found on the mesial or distal sides [3].

It has been reported that the prevalence of PEIR in the permanent dentition ranges from $0.5 \%$ to $3.5 \%$ depending on the type of radiographs used for detection [1,2]. Moreover, the most commonly affected teeth are the lower permanent second molars [2]. There is no clear link between PEIR and the child's gender, medical conditions, fluoride supplementation or systemic factors $[1,2,4]$. Despite this, some studies showed a strong association between PEIR and other developmental defects such as ectopic positioning and delay in dental development [2].

The exact etiology of PEIR remains inconclusive. However, the most acceptable theory is an idiopathic resorption by penetration of resorptive cells from the surrounding connective tissue [2,3]. Although PEIR defects are not carious in nature, once the tooth has emerged into the oral cavity, these defects are prone to bacterial colonization and development of dental caries $[1,2,5,6]$. Thus, Fissure sealant application was recommended by many authors to protect the tooth from bacterial colonization $[5,6]$.

The aim of this paper is to report a case of PEIR in the right mandibular second permanent molar of a young female patient, and to promote awareness of early detection of PEIR. Additionally, to emphasize the importance of periodic radiographic examination in monitoring the pattern of lesion progression.

\section{Case description}

An 8-year-old girl presented to the Pediatric Dentistry Clinic at King Faisal Specialist Hospital and Research Centre with her father in June 2012, for a comprehensive dental examination. She was with an unremarkable dental and medical history. The chief complaint was the difference in size between the upper front teeth. Clinical examination showed mixed dentition stage, multiple carious teeth, and multiple intact restorations done at a private clinic two years ago. Radiographic examination showed that the permanent upper right central incisor was impacted due to presence of a supernumerary tooth interfering with its eruption and that the primary right central incisor was overretained (Figure 1). An oral and maxillofacial surgeon was consulted and decision was made to perform surgical extraction of the impacted supernumerary tooth under general anesthesia.

Radiographic examination also showed that the developing lower right permanent second molar exhibited a caries-like radiolucent lesion (Figure 2A). The lesion was located in the distal part of the crown under the DEJ. The diagnosis of pre-eruptive intracoronal radiolucency was confirmed. Since the tooth was unerupted; the patient was asymptomatic; the decision was made to monitor the lesion

${ }^{\star}$ Correspondence to: Jawza H. Alfarraj, Pediatric Dentistry Department, King Faisal Specialist Hospital and Research, Riyadh Central 14215, Saudi Arabia, Email: Jawza500@gmail.com

Key words: Unerupted, resorption, radiolucency, coronal, dentinal, pediatric

Received: November 16, 2020; Accepted: November 23, 2020; Published: November 26, 2020 
by periodic panoramic radiographs to follow the progression of the lesion and root development. Consent was obtained from the father for reporting this case. Subsequently the patient was monitored clinically every three months and panoramic radiographs were taken annually. There is no obvious progression of the PEIR size or changes in location noted at age 10 and age 11 years old. (Figure 2BC).

At age of 12-year-old, clinically the PEIR-affected tooth had partially erupted; showing darkening of the distal fossa, mesial occlusal discolored groove with no cavitation, nor enamel defects (Figure 3). Radiographic examination revealed two-thirds of the root had developed. The lesion was in the same location, but was slightly larger than in previous radiographs (Figure 2D). Still, tooth was asymptomatic and there were no signs of pulpal involvement. Accordingly, decision was made to seal the tooth and monitor it until complete eruption for final treatment. The tooth was etched using 35\% phosphoric acid (Ultra-Etch, Ultradent Products, Inc., South Jordan, UT) then sealed with a resin-based fissure sealant (Concise Light Cure White Sealant, 3M ESPE, St. Paul, MN, USA).

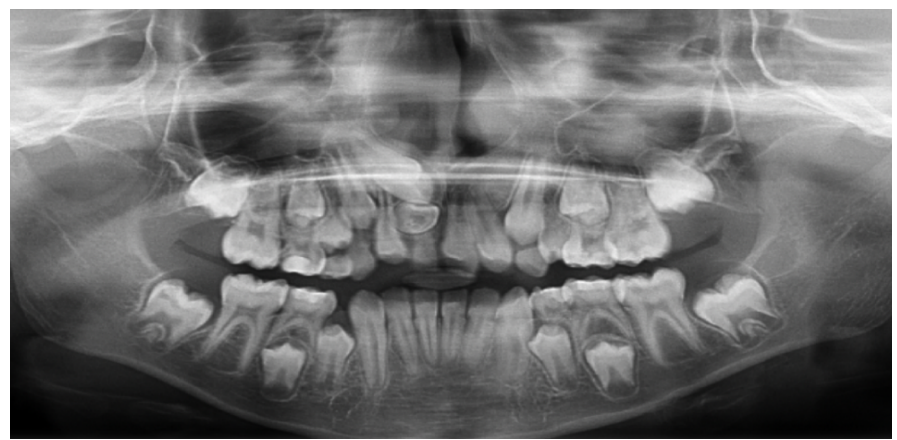

Figure 1. Panoramic radiographic examination shows impacted permanent upper right central incisor due to presence of a supernumerary tooth interfering with its eruption. Also, the primary right central incisor is overretained
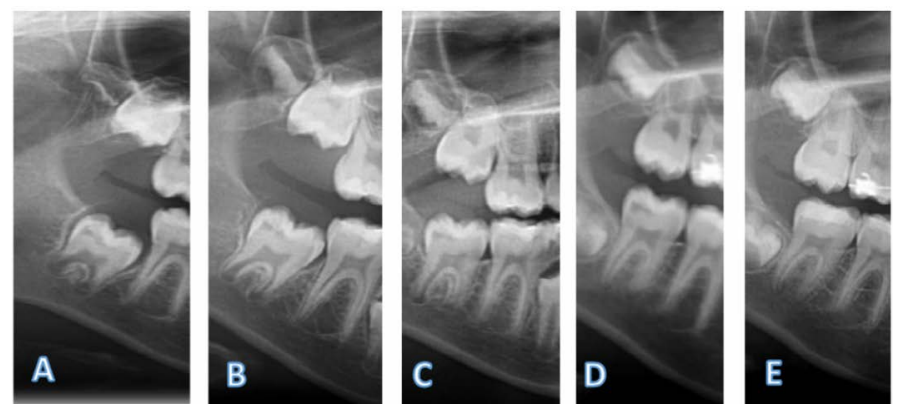

Figure 2. Annual series of panoramic radiographs taken for lesion progression monitoring. A. Baseline radiograph. B. At age 10 years old. C. At age 11 years old. D. At age 12 years old. E. One-year-follow up radiograph postoperatively

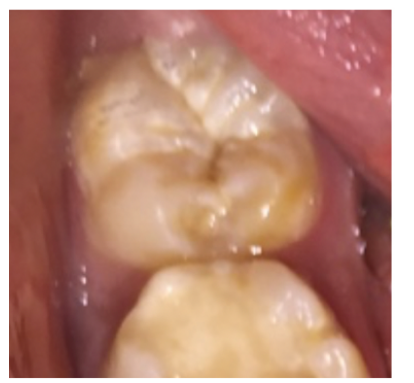

Figure 3. Clinical photograph of the PEIR-affected tooth shows tooth is partially erupted; has darkening of the distal fossa, mesial occlusal discolored groove with no cavitation nor enamel defects

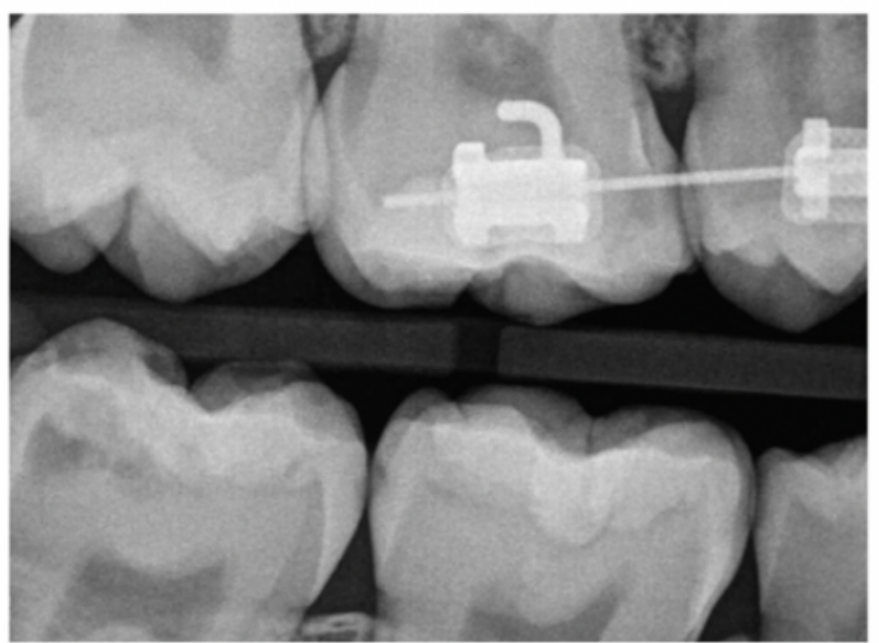

Figure 4. Bitewing radiograph of right side reveled a distally located radiolucency approximating the central fossa of tooth, with think dentin band separating the lesion from pulp

One year later, the PEIR-affected tooth had erupted completely. On clinical examination, the tooth was not tender to percussion with normal cold-test response. Radiographic examination of bitewing radiograph reveled a distally located caries-like radiolucency approximating the central fossa with thick dentin band separating the lesion from pulp (Figure 4). Under local anesthesia (2\% lidocaine with 1:100.000 epinephrine) and rubber dam application, the defective dentin was removed and the cavity was restored with shade A2 of Z100TM composite material (3M ESPE, St. Paul, MN). The patient was seen at the 6-month follow-up visit, and was asymptomatic. Clinical examination showed normal response to percussion and cold test. The restoration was intact with no discoloration or signs of marginal leakage. At one-year follow-up, she had the same clinical findings, and radiographic imaging revealed normal periapical area, periodontal ligament space, and bone trabeculation (Figure 2E).

\section{Discussion}

This case report describes the incidental finding of an asymptomatic PEIR in a permanent mandibular second molar of a female pediatric patient seen in a panoramic radiograph taken as a baseline for comprehensive examination.

Although the exact etiology remains uncertain, there are many suggested theories in the literature explaining this phenomenon [2]. It was thought that periapical inflammation of primary teeth is the main cause. Conversely, the most of PEIR defects were discovered within teeth with no primary predecessors [2]. Nonprogressive PEIR lesions are thought to be developmental in origin, produced by inclusion of uncalcified enamel matrix within the developing dentin [2,4]. However, progressive lesions are believed to result from idiopathic resorption $[3,7]$. Based on histological examination of PEIR lesions in extracted teeth, resorptive cells originating from the surrounding connective tissue of the developing tooth then initiate dentinal resorption [8]. Such lesions can result in almost complete destruction of the coronal dentin $[4,8]$. Therefore, PEIR lesions can be classified based on the progression pattern into two main types: nonprogressive (static) [2$3,9,10]$ and progressive (aggressive) [3,7-8].

Treatment options of PEIR-affected teeth differ depending on the progression pattern and size of lesion, as well as the presence of symptoms. In asymptomatic small nonprogressive lesions, many 
authors support the conservative approach, through monitoring until the tooth emerged into the oral cavity then restoring it $[2-3,10]$. Conversely symptomatic, or progressive, lesions need to be managed by a more aggressive approach including surgical exposure of the unerupted tooth and restoring it $[5,8]$.

Some case reports have shown that early management of progressive PEIR lesions will help in arresting the resorption process preventing further pulpal complications $[2,5]$. Thus, not only early detection of PEIR but also determining the progression pattern is important in treatment planning.

In this case, annual radiographic evaluation of the PEIR was sufficient to identify the lesion as a static nonprogressive type. However, there were no established guidelines on the periodicity of radiographic evaluation for PEIR lesions.

It is well known that PEIR-affected teeth are prone to posteruptive bacterial colonization and development of dental caries $[1,2,5,6]$. When a clinician decides to monitor a PEIR defect, the application of a fissure sealant to protect the tooth and prevent colonization is recommended $[5,6]$. Sealant can be placed either preeruptively or posteruptively [11]. In the literature, there is no studies yet exploring whether sealant placed preeruptively under surgical exposure is more effective. But we believe that if surgical exposure is planned, it is wise to consider restoring the tooth rather than just sealing it.

The case presented here had fissure-sealant placement after eruption, and the tooth was monitored over 14 months until full eruption. Removal of the defective dentinal tissue was followed by filling of the cavity with composite resin restoration. The bonding strength between resin composite restoration and the affected dentin has not been yet investigated, and we believe this is an area for future research. No clinical symptoms or radiographic findings were observed at the one-year follow-up, confirming short-term success of the treatment.

\section{Conclusion}

1. It is important to monitor the progression of asymptomatic PEIR lesions by periodic radiographic examination.

2. The timing of surgical intervention should be based on the pattern of lesion progression, presence of symptoms, and depth of lesion.

3. For the nonprogressive type of PEIR, we support following a conservative approach by monitoring until the tooth is fully erupted.

\section{References}

1. Seow WK (2000) Pre-eruptive intracoronal resorption as an entity of occult caries Pediatr Dent 22: 370-376. [Crossref]

2. Al-Batayneh OB, AlTawashi EK (2020) Pre-eruptive intra-coronal resorption of dentine: a review of aetiology, diagnosis, and management. Eur Arch Paediatr Dent 21: 1-11. [Crossref]

3. Currell SD, Cakar T (2019) Incidental observation of pre-eruptive intracoronal resorption after seven years. Aust Dent $J$ 64: 376-379.

4. Uzun I, Gunduz K, Canitezer G, Avsever H, Orhan KA (2015) A retrospective analysis of prevalence and characteristics of pre-eruptive intracoronal resorption in unerupted teeth of the permanent dentition: a multicentre study. Int Endod J 48: 1069-1076. [Crosserf]

5. Spierer WA, Fuks AB (2014) Pre-eruptive intra-coronal resorption: controversies and treatment options. J Clin Pediatr Dent 38: 326-328. [Crossref]

6. Czarnecki G, Morrow M, Peters M, Hu J (2014) Pre-eruptive intracoronal resorption of a permanent first molar. $J$ Dent Child 81: 151-155.

7. Umansky M, Tickotsky N, Friedlander-Barenboim S, Faibis S, Moskovitz M (2016) Age Related Prevalence of Pre-Eruptive Intracoronal Radiolucent Defects in the Permanent Dentition. J Clin Pediatr Dent 40: 103-106. [Crossref]

8. Singer S, Abbott PV, Booth DR (1991) Idiopathic coronal radiolucencies in unerupted permanent teeth. Case reports. Aust dent J 36: 32-37. [Crossref]

9. Counihan KP, O'Connell AC (2012) Case report: pre-eruptive intra-corona radiolucencies revisited. Eur Arch Pediat Dent 13: 221-226. [Crossref]

10. Moskovitz M, Holan G (2004) Pre-eruptive intracoronal radiolucent defect: a case of a nonprogressive lesion. J Denti Child 71: 175-178. [Crossref]

11. Czarnecki G, Morrow M, Peters M, Hu J (2014) Pre-eruptive intracoronal resorption of a permanent first molar. $J$ Dent Child $81: 151-5$.

Copyright: (C2020 Alfarraj JH. This is an open-access article distributed under the terms of the Creative Commons Attribution License, which permits unrestricted use, distribution, and reproduction in any medium, provided the original author and source are credited. 